OPEN ACCESS

Edited by:

Janos G. Filep,

Université de Montréal,

Canada

Reviewed by:

Panagiotis F. Christopoulos, Oslo University Hospital, Norway Martin Himly,

University of Salzburg, Austria Paola Italiani,

National Research Council (CNR), Italy

*Correspondence:

Yang Li

yang.li@siat.ac.cn

${ }^{t}$ These authors have contributed equally to this work and share first authorship

Specialty section: This article was submitted to

Molecular Innate Immunity,

a section of the journal

Frontiers in Immunology

Received: 28 April 2021 Accepted: 14 September 2021

Published: 04 October 2021

Citation:

Song Q, Javid A, Zhang G and Li Y (2021) Applications of Magnetite Nanoparticles in Cancer Immunotherapies: Present Hallmarks and Future Perspectives.

Front. Immunol. 12:701485. doi: 10.3389/fimmu.2021.701485

\section{Applications of Magnetite Nanoparticles in Cancer Immunotherapies: Present Hallmarks and Future Perspectives}

\author{
Qingle Song ${ }^{1 \dagger}$, Amaneh Javid ${ }^{1,2 \dagger}$, Guofang Zhang ${ }^{1}$ and Yang $\mathrm{Li}^{1 *}$ \\ 1 Laboratory of Immunology and Nanomedicine, Shenzhen Institute of Advanced Technology, Chinese Academy of Sciences, \\ Shenzhen, China, ${ }^{2}$ Department of Biological Sciences, Faculty of Applied Science and Engineering, Science and Arts \\ University, Yazd, Iran
}

Current immuno-oncotherapeutic protocols that inhibit tumor immune evasion have demonstrated great clinical success. However, the therapeutic response is limited only to a percentage of patients, and the immune-related adverse events can compromise the therapeutic benefits. Therefore, improving cancer immunotherapeutic approaches that pursue high tumor suppression efficiency and low side effects turn out to be a clinical priority. Novel magnetite nanoparticles (MNPs) exhibit great potential for therapeutic and imaging applications by utilizing their properties of superparamagnetism, good biocompatibility, as well as the easy synthesis and modulation/functionalization. In particular, the MNPs can exert magnetic hyperthermia to induce immunogenic cell death of tumor cells for effective antigen release and presentation, and meanwhile polarize tumorassociated macrophages (TAMs) to M1 phenotype for improved tumor killing capability, thus enhancing the anti-tumor immune effects. Furthermore, immune checkpoint antibodies, immune-stimulating agents, or tumor-targeting agents can be decorated on MNPs, thereby improving their selectivity for the tumor or immune cells by the unique magnetic navigation capability of MNPs to promote the tumor killing immune therapeutics with fewer side effects. This mini-review summarizes the recent progress in MNP-based immuno-oncotherapies, including activation of macrophage, promotion of cytotoxic $T$ lymphocyte (CTL) infiltration within tumors and modulation of immune checkpoint blockade, thus further supporting the applications of MNPs in clinical therapeutic protocols.

Keywords: magnetite nanoparticles, macrophages, cytotoxic T lymphocytes, immune checkpoint blockade, cancer immunotherapy

\section{INTRODUCTION}

Immuno-oncotherapy aims to activate the patient's immune system to recognize and kill tumor cells (1). Immunotherapeutic approaches date back to ancient Egypt, with the observation that tumors could be cured with bacterial infections, an event that we now attribute to immune activation. The modern immunotherapy of cancer started with Willian B. Coley at the end of the 
19th century with his "Coley's toxin", a mixture of killed bacteria, which non-specifically activated the immune responses, thereby resulting in tumor regression. Coley's toxin is still used in clinical trials, and pharmaceutical companies are interested in developing modern versions of it $(2,3)$. Current immunotherapies include several approaches for the nonspecific stimulation of innate immune mechanisms on the same line as Coley's toxin (such as the use of BCG in bladder cancer) (4), and strategies aiming at triggering specific antitumor immunity. These include the development of cancer vaccines, adoptive cell therapy, and checkpoint inhibitors (5). Indeed, immuno-oncotherapy based on immune checkpoint inhibitors has revolutionized the therapy of advanced and metastatic tumors, leading to an unprecedented rate of cure (6). However, all these therapies need further development and optimization. For instance, non-specific immunological stimulation is often associated with substantial inflammation (the Coley's toxin therapy was dubbed the "fever therapy"), and the therapy with checkpoint inhibitors that releases the block of immunity leads to uncontrolled reactions that can substantially affect the patient's own organism and causes the unwanted immune-related adverse events (irAEs) (7). In addition, in particular for immuno-oncotherapies aiming at activating specific anti-tumor immunity, the therapeutic approach is effective only for a limited number of patients, which depended on the degree of the immunogenicity for various tumor types (8). In an effort to improve the efficacy and safety of the immuno-oncotherapy, many studies have focused on the use of engineered nanoparticles (NPs) (9-12). In this context, magnetite nanoparticles (MNPs) are already in use for medical purposes, e.g., in iron replacement therapy and magnetic resonance imaging (MRI), approved by the US Food and Drug Administration (FDA) $(13,14)$. The European Medicines Agency (EMA) has also approved the use of iron oxide NPs $\left(\right.$ NanoTherm ${ }^{\circledR}$ ) for the treatment of intermittent glioblastoma multiforme (15). The suitability of MNPs as drug delivery system is based on a number of promising properties, which include their good superparamagnetism and biocompatibility, the easy synthesis and surface modulation/functionalization, and the magnetic hyperthermia and navigation capability (16-18). Based on these characteristics, MNPs can be also applied for theranostics and have been studied for a wide number of new medical applications in oncotherapy. Thus, this brief minireview tries to report the recent promising applications of MNPs in cancer immunotherapies.

\section{MNPs IN TUMOR IMAGING}

Currently, FDA has approved various MNPs for clinical imaging, including Ferumoxide (19), Ferumoxtran-10 (20, 21), Ferumoxsil (22), Ferucarbotran (23), Ferumoxytol (24, 25), and Magtrace (26). However, some of these MNPs have a low performance-price ratio, and some have been withdrawn from the market because of poor clinical results. Therefore, an increasing number of studies have investigated the optimized
MNPs to improve the imaging efficiency. Bai et al. (27) reported that the modification with a tumor-targeting peptide (cRGD, a molecule targeting the integrin $\alpha v \beta 3$ overexpressed by endothelium cells of angiogenic tumor vessels) on MNPs could facilitate the effective accumulation of MNPs into the tumor of mice, thus improving the efficiency on MRI application. Moreover, MNPs can be conjugated with other imaging components to realize multi-modal imaging (28). Li et al. (29) incorporated superparamagnetic iron oxide (SPIO) NPs into 1,2distearoyl-sn-glycero-3-phosphoethanolamine-N-[amino(polyethylene glycol)-5000] (DSPE-PEG5k) nanomicelles, which were further conjugated with a near-infrared fluorescence dye (Cy5) and the tumor-targeting peptide bombesin (Bom, used to target the overexpressed $G$ protein-coupled receptors in various malignancies). These nanomicelles could target mouse MDAMB-231 breast cancer and perform both MRI by the inner SPIO as well as the near-infrared fluorescence imaging by the Cy5 dye (29). These promising outcomes of MNPs in tumor imaging strategies demonstrated their advantages of easy surface modification and functionalization, and these properties could be further integrated into immuno-oncotherapeutic strategies. Recent studies of exploiting MNPs to enhance the efficacy of immunotherapy will be highlighted and discussed in the following paragraphs (30).

\section{MNPs FOR ENHANCING MACROPHAGE ANTI-TUMOR ACTIVITY}

Macrophages are the very important component of the innate immune system and first-line defense cells against pathogens and cancers (31). Macrophages are both surveillance and scavenging cells that patrol the tissue and recognize/eliminate senescent, anomalous, and dead cells, and activate effector cells to kill, ingest, and degrade pathogens and tumor cells after having initiated an inflammatory process. The activities of macrophages are mainly influenced by the developmental origin, tissue of residence, and acute microenvironmental cues (32). Although the capacity as antigen-presenting cells (APCs) is weaker than the dendritic cells (DCs), tumor-associated macrophages (TAMs) could re-activate primed $\mathrm{T}$ cells by cross-presenting tumor antigens in the tumor microenvironment (TME) rather than in the draining lymph node, thereby contributing to the adaptive immunity in addition to their main role in innate immune responses (33). Besides, the tumorkilling functionality of TAMs should also be ideally achieved through various mechanisms, e.g., the phagocytosis of tumor cells, secretion of cytokines (e.g., IFN $\gamma$ and TNF $\alpha$ ), production of inducible nitric oxide synthase, and induction of anti-tumor inflammation $(33,34)$. Unfortunately, phagocytosis is partially deactivated owing to the developed escaping mechanism of overexpressing "do-not-eat-me" signal on tumors (35). Furthermore, the most representative TAMs are pro-tumorigenic M2-like phenotype cells, which suppress the anti-tumor capacity of the M1-like phenotype cells, and contribute to the tumor growth, tumor immune evasion, and metastasis (36). Therefore, a bunch of investigations that aim to promote the phagocytosis and the M1 
polarization of the TAMs by the application of MNPs have been undertaken.

The expression of the cell surface integrin-associated protein CD47 (ubiquitously expressed on normal cells) is abundantly expressed on most tumor cells and creates a "do-not-eat-me" signal through binding with the signal-regulatory protein alpha (SIRP $\alpha$ ) on macrophages (37-39). SIRP $\alpha$ is a regulatory membrane glycoprotein expressed on the macrophage membrane that accumulates at a phagocytic synapse between macrophages and tumor cells upon CD47 binding $(37,40)$. Thus, the blockade of the CD47-SIRP $\alpha$ interaction by anti-CD47 antibody could restore the macrophagemediated phagocytosis against tumor (Figure 1) (41-43). Accordingly, a combined immunotherapeutic approach was proposed by using indocyanine green and sepantronium bromide co-loaded mesoporous silica NPs for photothermal and chemotherapy with primary tumors. This approach induced a primary tumor destruction that facilitated tumor antigen release and presentation for specific cytotoxic T lymphocyte (CTL) generation (44). Furthermore, MNPs coated by a silica layer conjugated with anti-CD47 antibody were subsequently magnetically guided to be accumulated at metastatic tumor sites to block the interaction of tumor-expressed CD47 with macrophages. As a consequence, macrophages recognized and destroyed/ phagocytosed metastatic tumor cells in synergy with activated CTLs (44). In addition, the Fc portion of some CD47 antibody could also drive macrophage for the antibody-dependent cellular phagocytosis (ADCP), which could further strengthen the macrophage-mediated anti-tumor activity (45). However, since CD47 is an essential molecule for protecting normal cells from macrophage attack, strategies for CD47 inhibition need to be very precisely targeted to tumor cells in order to avoid severe collateral damage, as observed with anti-CD47 antibody infusion that causes thrombocytopenia or anemia $(35,45,46)$.

The use of plasma membrane-coated biomimetic NPs was applied for a precise inhibition of tumor CD47 binding to macrophages $(47,48)$. The MNPs could be coated with membrane from genetically engineered cells that overexpressed a $\operatorname{SIRP} \alpha$ variant for CD47 binding and showed a 50,000-fold increased binding affinity. These biomimetic MNPs efficiently accumulated in the TME under external magnetic field guidance and specifically blocked the macrophage-inhibiting CD47-SIRP $\alpha$ binding between tumor cells and macrophages (49). Such magnetic navigation approach with MNPs decreases the risk of inducing severe side effects through enhanced tumor targeting. In addition, it is known that the TME can bias the infiltrating TAMs towards a tumor-promoting M2-like phenotype (50), thereby inhibiting the cytocidal and antigen-presenting capacity of M1 macrophages (51). There are evidences that MNPs could promote the re-polarization of TAMs towards an M1 functional phenotype, which resulted in the activation of the macrophage for tumor killing $(49,52)$. Ferumoxytol, one of the FDA-approved MNPs, was found able to upregulate the expression of M1-related genes (CD86 and TNFA) and to decrease expression of M2-related genes (CD206 and IL10) in a murine macrophage-like leukemia cell line in vitro (52). In vivo, experimental tumors were restricted in their growth by treatment with ferumoxytol and showed an increased number of M1-like infiltrating macrophages in comparison to the untreated tumors (52). Other studies also exploited MNPs for inducing M1 polarization of TAMs, as recently shown with polymeric NPs coated with a membrane of LPS treated macrophage, and encapsulating $\mathrm{Fe}_{3} \mathrm{O}_{4} \mathrm{NPs}$ and the imiquimod (Toll-like receptor 7 agonist, a strong macrophage activator), which were able to polarize TAMs towards M1 and achieve a concomitant restriction of experimental tumor growth (53). The membrane was prepared by the LPS stimulated macrophages to specifically target TAMs without direct M1 polarization effects that can be verified from the macrophage polarization results of PLGA-ION (PI) and membrane-coated PLGA-ION (PI@M) NPs. The CD80 expression in PI was 67.55\% vs. 74.31\% in PI@M. The higher M1 polarization of PI@M may be attributed to the enhanced internalization caused by the coated membrane camouflage and targeting capacity. Furthermore, vitamin $\mathrm{C}(\mathrm{Vc})$ was applied to eliminate the polarization capacity induced from the ROS pathway of enhancing p300/CBP acetyltransferase activity and promoting p53 acetylation by iron overload (54). Despite the fact that the ROS level of Vc+PI@M was reduced to 0.69-fold compared to the sole
A

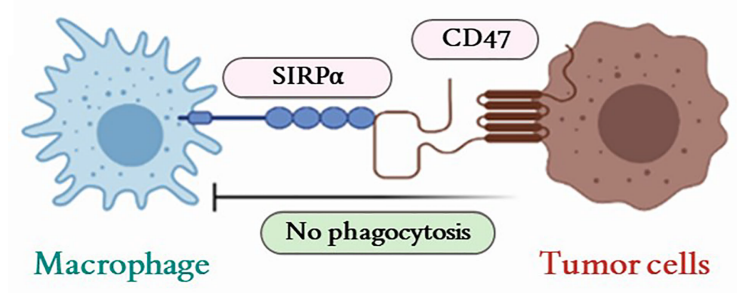

B

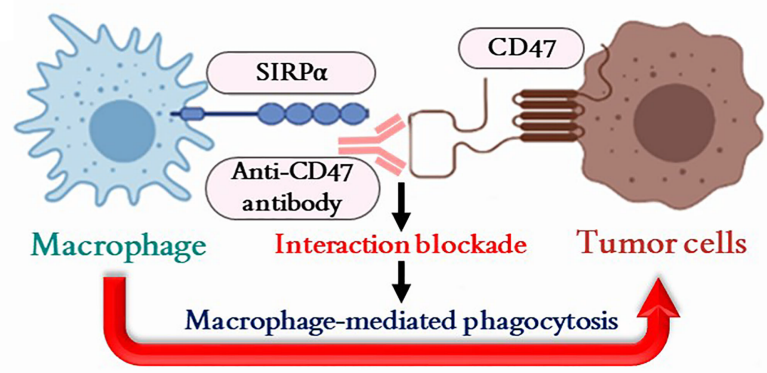

FIGURE 1 | Macrophage-mediated phagocytosis regulated by the CD47-SIRP $\alpha$ pathway. (A) The CD47-SIRP $\alpha$ interaction inhibits macrophage-mediated phagocytosis on tumor cells. (B) The interaction of CD47-SIRP $\alpha$ can be blocked by the anti-CD47 antibody to restore the macrophage-mediated phagocytosis against tumor cells. SIRP $\alpha$, signal-regulatory protein alpha; CD47, cluster of differentiation 47. 
PI@M treatment, CD80 expression in PI@M (74.31\%)and Vc+PI@ $\mathrm{M}(73.24 \%)$ was still in a similar range, indicating that the M1 polarization was mainly caused by MNPs rather than the ROS byproduct. Besides, a recent study indicated that, after taking a large amount of iron, macrophages could be polarized to M1 type by inhibiting the ERK phosphorylation (55). However, this mechanism still needs to be further investigated and confirmed critically.

Thus, MNPs have proven useful for enhancing the macrophage anti-tumor activity, both in inducing the M1 repolarization of TAMs and as carriers of inhibitors on tumor CD47 "do-not-eat-me" signal. Although these preliminary results are promising, future research will have to overcome several issues before successfully using MNPs as macrophage activators in cancer immunotherapy. These encompass the heterogeneity of MNP effects on macrophages (56) [may show no activation effects on human primary cells (57)], the need for very accurate tumor targeting to avoid the risk of severe off-target autoimmune and self-destructive effects (which is also the major current problem of immunotherapy with checkpoint inhibitors), the need to reach distant metastatic tumors, and the assessment of efficacy in non-solid tumors.

\section{MNPs FOR TURNING "COLD" TUMOR TO "HOT"}

Besides macrophages and the innate immune cells, stimulating CTLs could be considered as another effective approach for improving the anti-tumor immune responses and was also widely studied (58). A solid tumor can be defined as "cold" or "hot", depending on the degree and localization of tumorinfiltrating CTLs and the immunological condition of the TME $(59,60)$. The "cold" tumor exhibits an immune suppressive TME with an inadequate CTL infiltration and the excessively activated immunosuppressive cells, including regulatory $\mathrm{T}$ cells (Tregs), myeloid-derived suppressor cells (MDSCs), and TAMs (61). Meanwhile, the exhaustion of infiltrated CTLs can be induced by the immune suppressive checkpoints in TME, e.g., the programmed cell death protein 1 (PD-1)/programmed cell death ligand 1 (PD-L1) or the cytotoxic T lymphocyte antigen4 (CTLA-4), which hindered the development of anti-tumor specific CTLs responses (62). Conversely, a "hot" tumor exhibits a high accumulation and infiltration of non-exhausted CTLs, DCs, and natural killer cells (NKs), thus being an ideal target for immuno-oncotherapy $(58,63)$. Thus, to improve the overall response rate (ORR) of the current immuno-oncotherapy, a growing number of MNP-based immunotherapeutic strategies aim at turning the "cold" tumors to "hot" by enhancing the number of infiltrating CTLs and modulation of immune checkpoint blockade (ICB) in TME (Figure 2).

\section{MNPs Improving CTL Tumor Infiltration}

In the presence of an alternating magnetic field, MNPs can generate a magnetic hyperthermia by converting the magnetic energy into thermal energy, which can provoke immunogenic/ inflammatory cell death in the tumor $(64,65)$. The hyperthermia may induce immunogenic cell death (ICD) to release tumorassociated neoantigens and danger-associated molecular patterns (DAMPs), including the exposure of calreticulin (CRT), or the release of chromatin-binding protein high mobility group B1 (HMGB1) and the adenosine triphosphate (ATP) (66). Subsequently, the recognition and processing of the neoantigens can be promoted and followed with an effective Tcell priming (67). Thus, after the primary tumor elimination by the magnetic hyperthermia of the ferromagnetic iron oxide nanorings, the "eat-me" signal of CRT was elicited on the surface of the immunogenic dying 4T1 tumor cells and promoted the macrophage activation, which further enhanced the activation and infiltration of CTLs in the re-challenged or metastatic tumor on mice (68). Besides, as the most powerful APCs, the activation of DCs is crucial for promoting the infiltration of CTLs. To achieve a more effective tumor antigen uptaken by DCs, an MNP-based vaccine has been developed using $\mathrm{Fe}_{3} \mathrm{O}_{4}$ nanocluster core loading with the $\mathrm{CpG}$ oligodeoxynucleotide (CpG-ODN, a Toll-like receptor 9 agonist) and tumor cell membrane shell decorated with anti-CD205 for preferentially DC recognition (69). These DC targeting MNPs could extend the retention in lymph nodes by the magnetic control for an increased DC internalization. The CpG-ODN and the various antigens on the tumor cell membrane paved the way of DC maturation, thus facilitating the MHC cross-presentation and T-cell activation, eliciting a tremendous amount of CTL infiltration and responses in the tumor (69). Besides promoting the activation of DCs and CTLs, the possibility of binding MNPs on the surface of CTLs to improve the tumor infiltration by a magnetic field near the tumor site was also explored. By coating $\mathrm{Fe}_{3} \mathrm{O}_{4}$ nanoclusters with the leucocyte membranes modified with the co-stimulatory ligand CD28 antibody $(\alpha \mathrm{CD} 28)$ and peptide (SIINFEKL)-loaded major histocompatibility complex class-I (pMHC-I), the artificial antigen-presenting cells (aAPC) were constructed that successfully targeted CTLs for stimulation (70). Then, the CTLs that bound these nanoclusters could be effectively directed to tumor with an increased accumulation and infiltration facilitated upon magnetic guidance, resulting in an enhanced tumor killing efficiency (70).

\section{MNPs Modulating Immune Checkpoint Blockade}

Based on the current progress in immuno-oncotherapy, ICB therapy, e.g., PD-1/PD-L1 and CTLA-4, has exerted a major therapeutic effect on patients with advanced cancers (71). Immune checkpoints exhibit crucial functions in adjusting the balance of immune homeostasis and preventing T-cell-mediated autoimmune diseases, while they are also found hijacked by the tumor for suppressing the anti-tumor immune responses (72, 73). The PD-1 on T cells can be activated by binding its ligand PD-L1 on tumors, resulting an exhausted condition of infiltrated CTLs. CTLA-4, a new immunoglobulin superfamily candidate on T cells (including CD4, CD8, and Treg cells), restrains the costimulatory signals of CD28-CD80/86 by competitively binding with CD80/86 on antigen-presenting cells $(65,74)$. In clinical 


\section{Cold Hot}

A Magnetic hyperthermia

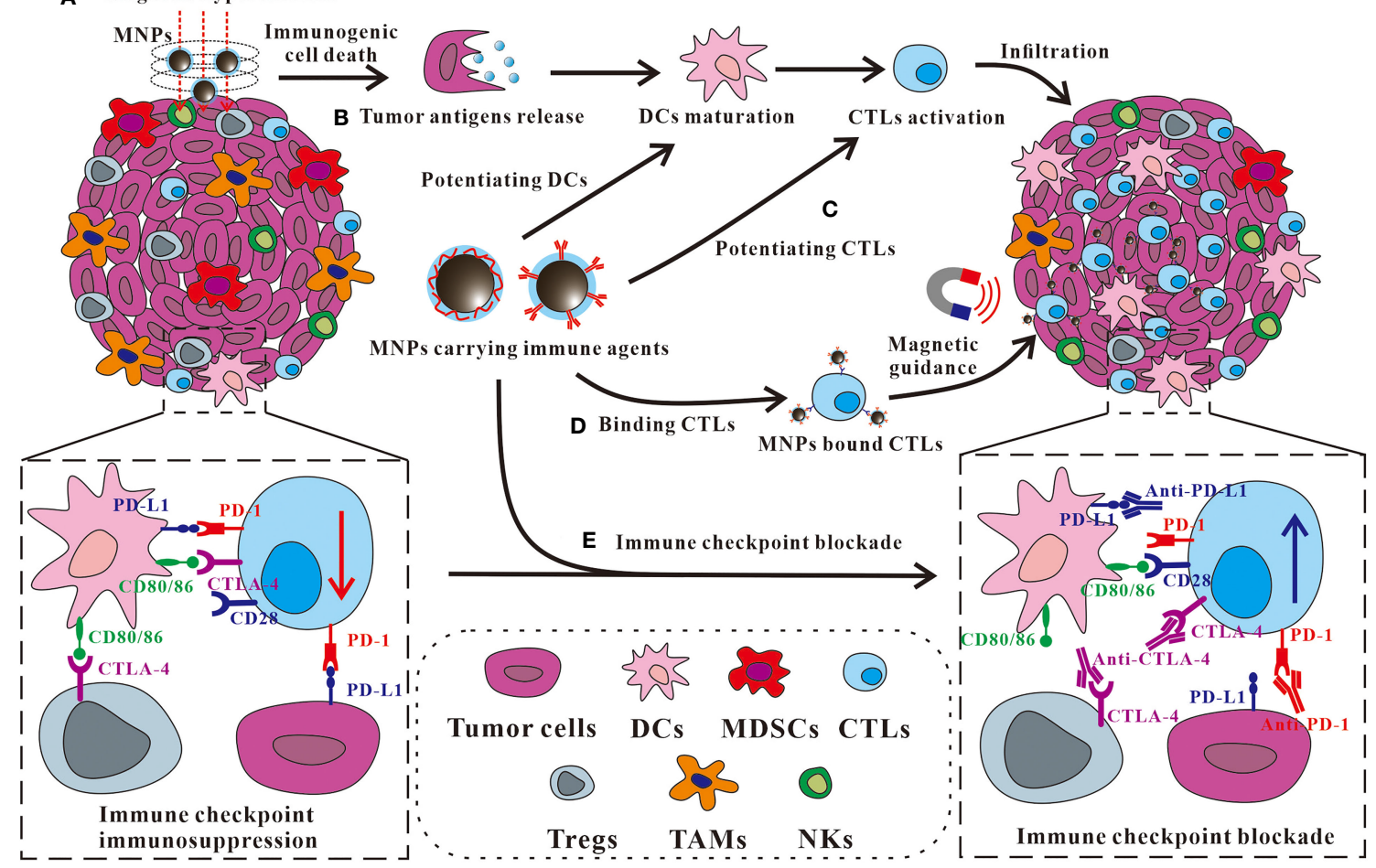

FIGURE 2 | Some representative approaches of MNPs to turn a "cold" tumor into "hot". (A) MNP-based magnetic hyperthermia ablation therapy is capable of inducing tumor immunogenic cell death to promote the release of antigen for DC maturation, and the subsequent CTL activation and infiltration in the tumor. (B, C) MNPs carrying immune agents such as agonists can be applied (B) for potentiating DCs or (C) for potentiating CTLs. (D) MNPs carning immune agents such as antibodies can bind CTLs, and direct them to the tumor with an improved accumulation and infiltration by the magnetic guidance. (E) The tumor accumulation of the immune checkpoint antibody can be enhanced by the conjugation on MNPs for an improved immunosuppressive pathway modulation with fewer adverse effects. DCs, dendritic cells; MDSCs, myeloid-derived suppressor cells; CTLs, cytotoxic T lymphocytes; Tregs, regulatory T cells; TAM, tumor-associated macrophages; NKs, natural killer cells; PD-1, programmed cell death protein 1; PD-L1, programmed cell death ligand 1; CTLA-4, cytotoxic T Iymphocyte-associated protein 4.

practice, anti-PD-1/PD-L1 and anti-CTLA-4 therapy proved effective for restoring the anti-tumor T-cell-mediated immune responses (75). However, ICB therapy only shows limited ORR with commonly associated irAEs, which limited its clinical application (76). Therefore, the combinational immunooncotherapy based on the multi-functional MNPs attracts considerable attention because of the immuno-stimulation effects of magnetic hyperthermia and the drug delivery capability of immuno-stimulatory adjuvant or checkpoint antibodies for the improved activation of CTLs and the accumulation of ICB antibodies (77).

The magnetic hyperthermia induced by MNPs could cause tumor ablation and the release of neoantigens. With the help of imiquimod (a toll-like receptor 7 agonist), CTLs were then effectively activated $(78,79)$. Thus, a further combinational approach by applying immune checkpoint antibodies, such as anti-CTLA-4 for Treg suppression or anti-PD-1/PD-L1 to restore exhausted CTLs, could be used for the synergistic oncotherapy to eliminate primary tumor and inhibit tumor metastasis, or even to generate a robust immunological memory for tumor recurrence prevention without noticeable systemic toxicity $(78,80)$. Beside the combination with checkpoint inhibitors separately, conjugating checkpoint inhibitors on MNPs demonstrated a better tumor targeting and treatment efficiency. The checkpoint inhibitor (anti-PD-L1) and CTL activators (anti-CD3 and anti-CD28) could be conjugated on the fucoidan-dextran-coated iron oxide NPs for an improved tumor accumulation by the magnetic guidance (81). These MNPs mediated ICB therapy-enhanced PD-L1 blockade and promoted CTL activation that prolonged the median survival time of tumor-bearing mice from 32 to 63 days with minimized adverse events owing to a much lower anti-PD-L1 dosage used than the soluble anti-PD-L1 treatment (81). Remarkably, the superparamagnetic iron nanoclusters were armed with PD-1 antibody by TME $\mathrm{pH}$-sensitive bond and successfully bound on CTLs in vitro. These MNP/CTL cells could be magnetically guided to the solid tumors in mice for improved CTL infiltration and tumor killing, and further aided with PD-1 antibody release through acid-mediated MNPs, for a synergistically enhanced immuno-oncotherapy with minimal side effects (82).

As these studies indicated, magnetic hyperthermia therapy induced by functionalized MNPs can effectively promote the 
immunological stimulation, tumor infiltration of CTLs, and the efficiency of ICB therapy. Moreover, various stimuli or ICB antibodies can be conjugated with the MNPs for improved targeting and immune cell activation in solid tumor. Meanwhile, the targeting cells, such as CTLs, attached with MNPs carrying ICB antibody on their surface, can be magnetically directed to tumor site for improved tumor accumulation. Such strategy has been proved successfully to enhance both CTL infiltration and ICB efficiency in one shot.

\section{CONCLUSIONS AND FUTURE PERSPECTIVES}

Taken together, extensive progresses have been made with various MNP platforms toward different applications for the enhanced immuno-oncotherapy. MNPs exhibited good capabilities for magnetic hyperthermia, magnetic navigation, and immuno-agent delivery to enhance macrophage anti-tumor capacity, to increase the tumor infiltration of CTLs, and to improve ICB efficiency. Moreover, the CTLs that adhered to the MNPs carrying immune checkpoint antibodies obtained the ability of magnetic navigation as well, which could greatly improve the CTL infiltration and ICB effects in solid tumors. These MNP-based immunotherapies exhibited higher anti-tumor efficiency with fewer side effects in the experimental studies. In the future therapeutic approaches of MNPs, the combination of these unique properties possessed by MNPs may be the most promising strategy for successful cancer therapeutics. The hyperthermia induced by MNPs can eliminate tumor cells directly, which provokes the ICD from DAMPs and antigen release for the improved anti-tumor immune responses. Moreover, the tumor tissue-retained MNPs could polarize the remaining TAMs to M1 macrophage as a further method to eliminate the residual tumor effectively. Consequently, the tumor metastasis and recurrence may be efficiently and sustainably inhibited by the synergistic strategy. Despite these advantages, disadvantages of high difficulty in mass production, lack of the precise analytic methods for examining the properties, and the complicated usage condition/equipment should be noticed for the future MNP application in oncotherapy. Moreover, in the clinical trials, only few MNP formulations, e.g., ferumoxytol, have shown new progress as an MRI-enhancing agent. Meanwhile, after the withdrawal of some MNPs in clinic, various regulations have currently been announced by the regulatory agencies, to assure a

\section{REFERENCES}

1. Steinman RM, Mellman I. Immunotherapy: Bewitched, Bothered, and Bewildered No More. Science (2004) 305(5681):197-200. doi: 10.1126/ science. 1099688

2. Hoption Cann SA, van Netten JP, van Netten C. Dr William Coley and Tumour Regression: A Place in History or in the Future. Postgrad Med J (2003) 79(938):672-80.

3. Karbach J, Neumann A, Brand K, Wahle C, Siegel E, Maeurer M, et al. Phase I Clinical Trial of Mixed Bacterial Vaccine (Coley's Toxins) in Patients With NYESO-1 Expressing Cancers: Immunological Effects and Clinical Activity. Clin Cancer Res (2012) 18(19):5449-59. doi: 10.1158/1078-0432.CCR-12-1116 safe, effective, and quality controllable MNPs for fundamental and translational development. With this concern, immense information needs to be researched to cope with the complex challenges, e.g., nano-bio interactions in the body, crossing physiological and tumor-specific technical hurdles, stealth behavior against late endosomal/lysosomal system into the tumor cytosol, and boosting the desired immunity without severe side effects or only with short-term adverse effects. Moreover, MNPs still have to face a number of biological barriers, such as the "Mononuclear Phagocyte System (MPS)", that abate the accumulation and localization of MNPs at the targeted tumor site, and limit their further application as efficacious immunotherapeutic vehicles. However, as promising outcomes have been seen from the current MNP synergetic immunooncotherapy, we believe that more potential approaches will be continuously exploited for ideal functionalized MNPs with successful clinical achievements.

\section{AUTHOR CONTRIBUTIONS}

YL conceived the idea of the review. QS and AJ performed the literature search, prepared the figures, and wrote the draft. GZ contributed to revising and editing the review. YL contributed to the critical revising, rewriting, and editing of the review. All authors contributed to the article and approved the submitted version.

\section{FUNDING}

This work was financially supported by the Shenzhen Science and Technology Program (GJHZ20190821155803877), the National Natural Science Foundation of China (32171390 and 31701005), the China Postdoctoral Science Foundation (2020M672898), the SIAT Innovation Program for Excellent Young Researchers (E1G068), and CAS President's International Fellowship Initiative (PIFI, 2020VBA0028, 2020VBA0022, 2022VBA0008 and 2021PB0060).

\section{ACKNOWLEDGMENTS}

We thank Prof. Diana Boraschi (Shenzhen Institute of Advanced Technology, Chinese Academy of Sciences) for her critical reading and helpful English editing of the manuscript.

4. Carlson RD, Flickinger JC, Snook AE. Talkin' Toxins: From Coley's to Modern Cancer Immunotherapy. Toxins (2020) 12(4):23. doi: 10.3390/ toxins 12040241

5. Yousefi H, Yuan J, Keshavarz-Fathi M, Murphy JF, Rezaei N. Immunotherapy of Cancers Comes of Age. Expert Rev Clin Immunol (2017) 13(10):1001-15. doi: 10.1080/1744666X.2017.1366315

6. Sharma P, Allison JP. The Future of Immune Checkpoint Therapy. Science (2015) 348(6230):56-61. doi: 10.1126/science.aaa8172

7. Naidoo J, Page DB, Li BT, Connell LC, Schindler K, Lacouture ME, et al. Toxicities of the Anti-PD-1 and Anti-PD-L1 Immune Checkpoint Antibodies. Ann Oncol (2015) 26(12):2375-91. doi: 10.1093/annonc/ mdv383 
8. Majidpoor J, Mortezaee K. The Efficacy of PD-1/PD-L1 Blockade in Cold Cancers and Future Perspectives. Clin Immunol (2021) 226:108707. doi: 10.1016/j.clim.2021.108707

9. Riley RS, June CH, Langer R, Mitchell MJ. Delivery Technologies for Cancer Immunotherapy. Nat Rev Drug Discov (2019) 18(3):175-96. doi: 10.1038/ s41573-018-0006-Z

10. Zhang Y, Zhang G, Chen L, Li Y. Modulation of the Inflammatory Tumor Microenvironment: A New Approach for Photothermal-Synergized Cancer Immunotherapy. Nanomed (Lond) (2019) 14(16):2101-4. doi: 10.2217/nnm2019-0156

11. Shao X, Ding Z, Zhou W, Li Y, Li Z, Cui H, et al. Intrinsic Bioactivity of Black Phosphorus Nanomaterials on Mitotic Centrosome Destabilization Through Suppression of PLK1 Kinase. Nat Nanotechnol (2021). doi: 10.1038/s41565021-00952-x

12. Zhao J, Lu D, Moya S, Yan H, Qiu M, Chen J, et al. Bispecific T-Cell Engager (BiTE) Immunotherapy of Ovarian Cancer Based on MIL-88a MOF/MC Gene Delivery System. Appl Materials Today (2020) 20:100701. doi: 10.1016/ j.apmt.2020.100701

13. Zeng Q, Li H, Jiang H, Yu J, Wang Y, Ke H, et al. Tailoring Polymeric Hybrid Micelles With Lymph Node Targeting Ability to Improve the Potency of Cancer Vaccines. Biomaterials (2017) 122:105-13. doi: 10.1016/ j.biomaterials.2017.01.010

14. Kasturi SP, Skountzou I, Albrecht RA, Koutsonanos D, Hua T, Nakaya HI, et al. Programming the Magnitude and Persistence of Antibody Responses With Innate Immunity. Nature (2011) 470(7335):543-7. doi: 10.1038/ nature09737

15. Zhu Q, Egelston C, Vivekanandhan A, Uematsu S, Akira S, Klinman DM, et al. Toll-Like Receptor Ligands Synergize Through Distinct Dendritic Cell Pathways to Induce T Cell Responses: Implications for Vaccines. Proc Natl Acad Sci USA (2008) 105(42):16260-5. doi: 10.1073/pnas.0805325105

16. López-Castro JD, Maraloiu AV, Delgado JJ, Calvino JJ, Blanchin MG, Gálvez $\mathrm{N}$, et al. From Synthetic to Natural Nanoparticles: Monitoring the Biodegradation of SPIO (P904) Into Ferritin by Electron Microscopy. Nanoscale (2011) 3(11):4597-9. doi: 10.1039/c1nr10980d

17. Cho NH, Cheong TC, Min JH, Wu JH, Lee SJ, Kim D, et al. A Multifunctional Core-Shell Nanoparticle for Dendritic Cell-Based Cancer Immunotherapy. Nat Nanotechnol (2011) 6(10):675-82. doi: 10.1038/nnano.2011.149

18. Trabulo S, Aires A, Aicher A, Heeschen C, Cortajarena AL. Multifunctionalized Iron Oxide Nanoparticles for Selective Targeting of Pancreatic Cancer Cells. Biochim Biophys Acta Gen Subj (2017) 1861 (6):1597-605. doi: 10.1016/j.bbagen.2017.01.035

19. Wang YX. Current Status of Superparamagnetic Iron Oxide Contrast Agents for Liver Magnetic Resonance Imaging. World J Gastroenterol (2015) 21 (47):13400-2. doi: 10.3748/wjg.v21.i47.13400

20. Bourrinet P, Bengele HH, Bonnemain B, Dencausse A, Idee JM, Jacobs PM, et al. Preclinical Safety and Pharmacokinetic Profile of Ferumoxtran-10, an Ultrasmall Superparamagnetic Iron Oxide Magnetic Resonance Contrast Agent. Invest Radiol (2006) 41(3):313-24. doi: 10.1097/01.rli.0000197669.80475.dd

21. Fortuin AS, Brüggemann R, van der Linden J, Panfilov I, Israël B, Scheenen TWJ, et al. Ultra-Small Superparamagnetic Iron Oxides for Metastatic Lymph Node Detection: Back on the Block. Wiley Interdiscip Rev Nanomed Nanobiotechnol (2018) 10(1):e1471. doi: 10.1002/wnan.1471

22. Wáng YX, Idée JM. A Comprehensive Literatures Update of Clinical Researches of Superparamagnetic Resonance Iron Oxide Nanoparticles for Magnetic Resonance Imaging. Quant Imaging Med Surg (2017) 7(1):88-122. doi: 10.21037/qims.2017.02.09

23. Reimer P, Balzer T. Ferucarbotran (Resovist): A New Clinically Approved RES-Specific Contrast Agent for Contrast-Enhanced MRI of the Liver: Properties, Clinical Development, and Applications. Eur Radiol (2003) 13 (6):1266-76. doi: 10.1007/s00330-002-1721-7

24. Trujillo-Alonso V, Pratt EC, Zong H, Lara-Martinez A, Kaittanis C, Rabie MO, et al. FDA-Approved Ferumoxytol Displays Anti-Leukaemia Efficacy Against Cells With Low Ferroportin Levels. Nat Nanotechnol (2019) 14 (6):616-22. doi: 10.1038/s41565-019-0406-1

25. Bullivant JP, Zhao S, Willenberg BJ, Kozissnik B, Batich CD, Dobson J. Materials Characterization of Feraheme/ferumoxytol and Preliminary Evaluation of its Potential for Magnetic Fluid Hyperthermia. Int J Mol Sci (2013) 14(9):17501-10. doi: 10.3390/ijms140917501
26. Pouw JJ, Ahmed M, Anninga B, Schuurman K, Pinder SE, Van Hemelrijck M, et al. Comparison of Three Magnetic Nanoparticle Tracers for Sentinel Lymph Node Biopsy in an In Vivo Porcine Model. Int J Nanomed (2015) 10:1235-43. doi: $10.2147 / \mathrm{IJN} . S 76962$

27. Bai C, Jia Z, Song L, Zhang W, Chen Y, Zang F, et al. Time-Dependent T1-T2 Switchable Magnetic Resonance Imaging Realized by C(RGDyK) Modified Ultrasmall Fe3O4 Nanoprobes. Advanced Funct Materials (2018) 28 (32):1802281.1-.9. doi: 10.1002/adfm.201802281

28. Shin TH, Choi Y, Kim S, Cheon J. Recent Advances in Magnetic NanoparticleBased Multi-Modal Imaging. Chem Soc Rev (2015) 44(14):4501-16. doi: $10.1039 / \mathrm{C} 4 \mathrm{CS} 00345 \mathrm{D}$

29. Li L, Wu C, Pan L, Li X, Kuang A, Cai H, et al. Bombesin-Functionalized Superparamagnetic Iron Oxide Nanoparticles for Dual-Modality MR/NIRFI in Mouse Models of Breast Cancer. Int J Nanomed (2019) 14:6721-32. doi: $10.2147 / \mathrm{IJN} . S 211476$

30. Wu X, Zhang H. Therapeutic Strategies of Iron-Based Nanomaterials for Cancer Therapy. BioMed Mater (2021) 16(3):032003. doi: 10.1088/1748$605 \mathrm{X} / \mathrm{abd} 0 \mathrm{c} 4$

31. Demaria O, Cornen S, Daëron M, Morel Y, Medzhitov R, Vivier E. Harnessing Innate Immunity in Cancer Therapy. Nature (2019) 574(7776):45-56. doi: 10.1038/s41586-019-1593-5

32. DeNardo DG, Ruffell B. Macrophages as Regulators of Tumour Immunity and Immunotherapy. Nat Rev Immunol (2019) 19(6):369-82. doi: 10.1038/ s41577-019-0127-6

33. Lecoultre M, Dutoit V, Walker PR. Phagocytic Function of Tumor-Associated Macrophages as a Key Determinant of Tumor Progression Control: A Review. J Immunother Cancer (2020) 8(2):e001408. doi: 10.1136/jitc-2020-001408

34. Najafi M, Hashemi Goradel N, Farhood B, Salehi E, Nashtaei MS, Khanlarkhani N, et al. Macrophage Polarity in Cancer: A Review. J Cell Biochem (2019) 120(3):2756-65. doi: 10.1002/jcb.27646

35. Feng M, Jiang W, Kim BYS, Zhang CC, Fu YX, Weissman IL. Phagocytosis Checkpoints as New Targets for Cancer Immunotherapy. Nat Rev Cancer (2019) 19(10):568-86. doi: 10.1038/s41568-019-0183-z

36. Pathria P, Louis TL, Varner JA. Targeting Tumor-Associated Macrophages in Cancer. Trends Immunol (2019) 40(4):310-27. doi: 10.1016/j.it.2019.02.003

37. Chao MP, Alizadeh AA, Tang C, Myklebust JH, Varghese B, Gill S, et al. AntiCD47 Antibody Synergizes With Rituximab to Promote Phagocytosis and Eradicate non-Hodgkin Lymphoma. Cell (2010) 142(5):699-713. doi: 10.1016/j.cell.2010.07.044

38. Majeti R, Chao MP, Alizadeh AA, Pang WW, Jaiswal S, Gibbs KDJr., et al. CD47 is an Adverse Prognostic Factor and Therapeutic Antibody Target on Human Acute Myeloid Leukemia Stem Cells. Cell (2009) 138(2):286-99. doi: 10.1016/j.cell.2009.05.045

39. Jaiswal S, Jamieson CH, Pang WW, Park CY, Chao MP, Majeti R, et al. CD47 is Upregulated on Circulating Hematopoietic Stem Cells and Leukemia Cells to Avoid Phagocytosis. Cell (2009) 138(2):271-85. doi: 10.1016/j.cell.2009.05.046

40. Rezaei G, Habibi-Anbouhi M, Mahmoudi M, Azadmanesh K, Moradi-Kalbolandi S, Behdani M, et al. Development of Anti-CD47 Single-Chain Variable Fragment Targeted Magnetic Nanoparticles for Treatment of Human Bladder Cancer. Nanomed (Lond) (2017) 12(6):597-613. doi: 10.2217/nnm-2016-0302

41. Lu Q, Chen X, Wang S, Lu Y, Yang C, Jiang G. Potential New Cancer Immunotherapy: Anti-CD47-Sirp $\alpha$ Antibodies. Onco Targets Ther (2020) 13:9323-31. doi: 10.2147/OTT.S249822

42. Matlung HL, Szilagyi K, Barclay NA, van den Berg TK. The CD47-Sirp $\alpha$ Signaling Axis as an Innate Immune Checkpoint in Cancer. Immunol Rev (2017) 276(1):145-64. doi: 10.1111/imr.12527

43. Chen Q, Wang C, Zhang X, Chen G, Hu Q, Li H, et al. In Situ Sprayed Bioresponsive Immunotherapeutic Gel for Post-Surgical Cancer Treatment Nat Nanotechnol (2019) 14(1):89-97. doi: 10.1038/s41565-018-0319-4

44. Zhang Y, Chen H, Wang H, Wang T, Pan H, Ji W, et al. A Synergistic Cancer Immunotherapy Nano-System for Preventing Tumor Growth. Chem Eng J (2020) 380:122472. doi: 10.1016/j.cej.2019.122472

45. Pietsch EC, Dong J, Cardoso R, Zhang X, Chin D, Hawkins R, et al. AntiLeukemic Activity and Tolerability of Anti-Human CD47 Monoclonal Antibodies. Blood Cancer J (2017) 7(2):e536. doi: 10.1038/bcj.2017.7

46. Advani R, Flinn I, Popplewell L, Forero A, Bartlett NL, Ghosh N, et al. CD47 Blockade by Hu5F9-G4 and Rituximab in Non-Hodgkin's Lymphoma. N Engl J Med (2018) 379(18):1711-21. doi: 10.1056/NEJMoa1807315 
47. Xia Q, Zhang Y, Li Z, Hou X, Feng N. Red Blood Cell Membrane-Camouflaged Nanoparticles: A Novel Drug Delivery System for Antitumor Application. Acta Pharm Sin B (2019) 9(4):675-89. doi: 10.1016/j.apsb.2019.01.011

48. Liu WL, Zou MZ, Liu T, Zeng JY, Li X, Yu WY, et al. Cytomembrane Nanovaccines Show Therapeutic Effects by Mimicking Tumor Cells and Antigen Presenting Cells. Nat Commun (2019) 10(1):3199. doi: 10.1038/ s41467-019-11157-1

49. Rao L, Zhao SK, Wen C, Tian R, Lin L, Cai B, et al. Activating MacrophageMediated Cancer Immunotherapy by Genetically Edited Nanoparticles. $A d v$ Mater (2020) 32(47):e2004853. doi: 10.1002/adma.202004853

50. Mantovani A, Longo DL. Macrophage Checkpoint Blockade in Cancer - Back to the Future. N Engl J Med (2018) 379(18):1777-9. doi: 10.1056/NEJMe1811699

51. Italiani P, Boraschi D. From Monocytes to M1/M2 Macrophages: Phenotypical vs. Functional Differentiation. Front Immunol (2014) 5:22. doi: 10.3389/fimmu.2014.00514

52. Zanganeh S, Hutter G, Spitler R, Lenkov O, Mahmoudi M, Shaw A, et al. Iron Oxide Nanoparticles Inhibit Tumour Growth by Inducing Pro-Inflammatory Macrophage Polarization in Tumour Tissues. Nat Nanotechnol (2016) 11 (11):986-94. doi: 10.1038/nnano.2016.168

53. Liu L, Wang Y, Guo X, Zhao J. Zhou S. A Biomimetic Polymer Magnetic Nanocarrier Polarizing Tumor-Associated Macrophages for Potentiating Immunotherapy. Small (2020) 16(38):e2003543 doi: 10.1002/smll.202003543

54. Zhou Y, Que K-T, Zhang Z, Yi ZJ, Zhao PX, You Y, et al. Iron Overloaded Polarizes Macrophage to Proinflammation Phenotype Through ROS/acetylP53 Pathway. Cancer Med (2018) 7(8):4012-22. doi: 10.1002/cam4.1670

55. Wang S, Cheng M, Peng P, Lou Y, Zhang A, Liu P. Iron Released After CryoThermal Therapy Induced M1 Macrophage Polarization, Promoting the Differentiation of CD4(+) T Cells Into CTLs. Int J Mol Sci (2021) 22 (13):7010. doi: 10.3390/ijms22137010

56. Della Camera G, Madej M, Ferretti AM, La Spina R, Li Y, Corteggio A, et al. Personalised Profiling of Innate Immune Memory Induced by Nano-Imaging Particles in Human Monocytes. Front Immunol (2021) 12(3053). doi: 10.3389/ fimmu.2021.692165

57. Ferretti AM, Usseglio S, Mondini S, Drago C, La Mattina R, Chini B, et al. Towards Bio-Compatible Magnetic Nanoparticles: Immune-Related Effects, in-Vitro Internalization, and in-Vivo Bio-Distribution of Zwitterionic Ferrite Nanoparticles With Unexpected Renal Clearance. J Colloid Interface Sci (2021) 582:678-700. doi: 10.1016/j.jcis.2020.08.026

58. Song Q, Zhang G, Wang B, Cao G, Li D, Wang Y, et al. Reinforcing the Combinational Immuno-Oncotherapy of Switching "Cold" Tumor to "Hot" by Responsive Penetrating Nanogels. ACS Appl Mater Interfaces (2021) 13 (31):36824-38. doi: 10.1021/acsami.1c08201

59. Chen DS, Mellman I. Elements of Cancer Immunity and the Cancer-Immune Set Point. Nature (2017) 541(7637):321-30. doi: 10.1038/nature21349

60. Liu Y-T, Sun Z-J. Turning Cold Tumors Into Hot Tumors by Improving TCell Infiltration. Theranostics (2021) 11(11):5365-86. doi: 10.7150/thno.58390

61. Hegde PS, Chen DS. Top 10 Challenges in Cancer Immunotherapy. Immunity (2020) 52(1):17-35. doi: 10.1016/j.immuni.2019.12.011

62. Pardoll DM. The Blockade of Immune Checkpoints in Cancer Immunotherapy. Nat Rev Cancer (2012) 12(4):252-64. doi: 10.1038/nrc3239

63. Hegde PS, Karanikas V, Evers S. The Where, the When, and the How of Immune Monitoring for Cancer Immunotherapies in the Era of Checkpoint Inhibition. Clin Cancer Res (2016) 22(8):1865-74. doi: 10.1158/1078-0432.CCR-15-1507

64. Liu X, Zhang Y, Wang Y, Zhu W, Li G, Ma X, et al. Comprehensive Understanding of Magnetic Hyperthermia for Improving Antitumor Therapeutic Efficacy. Theranostics (2020) 10(8):3793-815. doi: 10.7150/thno.40805

65. Zhang Y, Zhang G, Wang G, Wu L, Monteiro-Riviere NA, Li Y. The Synergistic Strategies for the Immuno-Oncotherapy With Photothermal Nanoagents. Wiley Interdiscip Rev Nanomed Nanobiotechnol (2021) 13(5): e1717. doi: 10.1002/wnan.1717

66. Sweeney EE, Cano-Mejia J, Fernandes R. Photothermal Therapy Generates a Thermal Window of Immunogenic Cell Death in Neuroblastoma. Small (2018) 14(20):e1800678. doi: 10.1002/smll.201800678

67. Krysko DV, Garg AD, Kaczmarek A, Krysko O, Agostinis P, Vandenabeele P. Immunogenic Cell Death and DAMPs in Cancer Therapy. Nat Rev Cancer (2012) 12(12):860-75. doi: 10.1038/nrc3380

68. Liu X, Zheng J, Sun W, Zhao X, Li Y, Gong N, et al. Ferrimagnetic Vortex Nanoring-Mediated Mild Magnetic Hyperthermia Imparts Potent
Immunological Effect for Treating Cancer Metastasis. ACS nano (2019) 13 (8):8811-25. doi: 10.1021/acsnano.9b01979

69. Li F, Nie W, Zhang F, Lu G, Lv C, Lv Y, et al. Engineering Magnetosomes for High-Performance Cancer Vaccination. ACS Cent Sci (2019) 5(5):796-807. doi: 10.1021/acscentsci.9b00060

70. Zhang Q, Wei W, Wang P, Zuo L, Li F, Xu J, et al. Biomimetic Magnetosomes as Versatile Artificial Antigen-Presenting Cells to Potentiate T-Cell-Based Anticancer Therapy. ACS Nano (2017) 11(11):10724-32. doi: 10.1021/ acsnano.7b04955

71. Khalil DN, Smith EL, Brentjens RJ, Wolchok JD. The Future of Cancer Treatment: Immunomodulation, CARs and Combination Immunotherapy. Nat Rev Clin Oncol (2016) 13(5):273-90. doi: 10.1038/nrclinonc.2016.25

72. Alsaab HO, Sau S, Alzhrani R, Tatiparti K, Bhise K, Kashaw SK, et al. PD-1 and PD-L1 Checkpoint Signaling Inhibition for Cancer Immunotherapy: Mechanism, Combinations, and Clinical Outcome. Front Pharmacol (2017) 8:561. doi: $10.3389 /$ fphar.2017.00561

73. Deng $\mathrm{H}$, Zhang Z. The Application of Nanotechnology in Immune Checkpoint Blockade for Cancer Treatment. J Control Release (2018) 290:28-45. doi: 10.1016/j.jconrel.2018.09.026

74. Intlekofer AM, Thompson CB. At the Bench: Preclinical Rationale for CTLA4 and PD-1 Blockade as Cancer Immunotherapy. J Leukoc Biol (2013) 94 (1):25-39. doi: 10.1189/jlb.1212621

75. Buchbinder E, Hodi FS. Cytotoxic T Lymphocyte Antigen-4 and Immune Checkpoint Blockade. J Clin Invest (2015) 125(9):3377-83. doi: 10.1172/ JCI80012

76. Byun DJ, Wolchok JD, Rosenberg LM, Girotra M. Cancer Immunotherapy Immune Checkpoint Blockade and Associated Endocrinopathies. Nat Rev Endocrinol (2017) 13(4):195-207. doi: 10.1038/nrendo.2016.205

77. Jin J, Zhao Q. Engineering Nanoparticles to Reprogram Radiotherapy and Immunotherapy: Recent Advances and Future Challenges. J Nanobiotechnol (2020) 18(1):75. doi: 10.1186/s12951-020-00629-y

78. Chao Y, Chen G, Liang C, Xu J, Dong Z, Han X, et al. Iron Nanoparticles for Low-Power Local Magnetic Hyperthermia in Combination With Immune Checkpoint Blockade for Systemic Antitumor Therapy. Nano Lett (2019) 19 (7):4287-96. doi: 10.1021/acs.nanolett.9b00579

79. Sobhani N, Tardiel-Cyril DR, Davtyan A, Generali D, Roudi R, Li Y. CTLA-4 in Regulatory T Cells for Cancer Immunotherapy. Cancers (Basel) (2021) 13 (6):1440. doi: 10.3390/cancers 13061440

80. Wang Z, Zhang F, Shao D, Chang Z, Wang L, Hu H, et al. Janus Nanobullets Combine Photodynamic Therapy and Magnetic Hyperthermia to Potentiate Synergetic Anti-Metastatic Immunotherapy. Adv Sci (Weinh) (2019) 6 (22):1901690. doi: 10.1002/advs.201901690

81. Chiang CS, Lin YJ, Lee R, Lai YH, Cheng HW, Hsieh CH, et al. Combination of Fucoidan-Based Magnetic Nanoparticles and Immunomodulators Enhances Tumour-Localized Immunotherapy. Nat Nanotechnol (2018) 13 (8):746-54. doi: 10.1038/s41565-018-0146-7

82. Nie W, Wei W, Zuo L, Lv C, Zhang F, Lu GH, et al. Magnetic Nanoclusters Armed With Responsive PD-1 Antibody Synergistically Improved Adoptive T-Cell Therapy for Solid Tumors. ACS Nano (2019) 13(2):1469-78. doi: 10.1021/acsnano.8b07141

Conflict of Interest: The authors declare that the research was conducted in the absence of any commercial or financial relationships that could be construed as a potential conflict of interest.

Publisher's Note: All claims expressed in this article are solely those of the authors and do not necessarily represent those of their affiliated organizations, or those of the publisher, the editors and the reviewers. Any product that may be evaluated in this article, or claim that may be made by its manufacturer, is not guaranteed or endorsed by the publisher.

Copyright (C) 2021 Song, Javid, Zhang and Li. This is an open-access article distributed under the terms of the Creative Commons Attribution License (CC BY). The use, distribution or reproduction in other forums is permitted, provided the original author(s) and the copyright owner(s) are credited and that the original publication in this journal is cited, in accordance with accepted academic practice. No use, distribution or reproduction is permitted which does not comply with these terms. 